\title{
Noninvasive Pulmonary Nodule Elastometry by CT and Deformable Image
}

\section{Registration}

\author{
Mohammadreza Negahdar, Ph.D.," Carolina E. Fasola, M.D., MPH," Amy S Yu, Ph.D.," Rie von \\ Eyben, M.S., Tokihiro Yamamoto, Ph.D., ${ }^{\S}$ Maximilian Diehn, M.D., Ph.D., Dominik Fleischmann, \\ M.D., ${ }^{\dagger}$ Lu Tian, Sc.D., ${ }^{\dagger}$ Billy W. Loo, Jr., M.D., Ph.D., Peter G. Maxim, Ph.D." \\ Departments of ${ }^{*}$ Radiation Oncology, Radiology, and Health Research and Policy, Stanford University, \\ Stanford, CA; ${ }^{\S}$ Department of Radiation Oncology, University of California, Davis, CA
}

\section{Corresponding author:}

Peter G. Maxim, Ph.D., Stanford Cancer Center, 875 Blake Wilbur Dr., Stanford, CA 94305. Tel: (650) 724-3018; Fax: (650) 725-8231; E-mail: pmaxim@stanford.edu

Billy W. Loo, Jr., M.D., Ph.D., Stanford Cancer Center, 875 Blake Wilbur Dr., Stanford, CA 94305. Tel: (650) 726-

7143; Fax: (650) 725-8231; E-mail: bwloo@stanford.edu

Running title: CT Pulmonary Nodule Elastometry

Acknowledgement: This study has been supported by Department of Defense LCRP 2011 \#W81XWH-12-1-0286.

Conflict of interest: None.

This paper has 12 pages, 4 figures, and 1 table. There is one figure and one table as supplementary data.

Keywords: Malignant Pulmonary Nodule (MPN), Elasticity, Deformable image registration (DIR), X-ray CT, Lung Cancer. 
Background and Purpose: To develop a noninvasive method for determining malignant pulmonary nodule (MPN) elasticity, and compare it against expert dual-observer manual contouring.

Methods and Materials: We analyzed breath-hold images at extreme tidal volumes of 23 patients with 30 MPN treated with stereotactic ablative radiotherapy. Deformable image registration (DIR) was applied to the breath-hold images to determine the volumes of the MPNs and a ring of surrounding lung tissue (ring) in each state. MPNs were also manually delineated on deep inhale and exhale images by two observers. Volumes were compared between observers and DIR by Dice similarity. Elasticity was defined as the absolute value of the volume ratio of the MPN minus one normalized to that of the ring.

Results: For all 30 tumors the Dice coefficient was $0.79 \pm 0.07$ and $0.79 \pm 0.06$ between DIR with observers 1 and 2, respectively, close to the inter-observer Dice value, $0.81 \pm 0.1$. The elasticity of MPNs was $1.24 \pm 0.26$, demonstrating that volume change of the MPN was less than that of the surrounding lung.

Conclusion: We developed a noninvasive CT elastometry method based on DIR that measures the elasticity of biopsy-proven MPN. Our future direction would be to develop this method to distinguish malignant from benign nodules. 


\section{Introduction}

Elastometry provides tissue characterization that may help distinguish malignant from benign tissues, in contrast to static morphological imaging, which cannot provide this degree of information [1]. Several modalities of elasticity imaging, mostly based on ultrasound have been proposed and applied to a number of clinical applications including breast carcinoma [2,3], metastatic melanoma [4,5], head and neck carcinoma [6], and colorectal carcinoma [2]. However, since air is opaque in ultrasound images, this imaging modality has been considered insufficient for adequately visualizing lung tissue. Magnetic resonance elastography is a MRI-based technique which is capable of assessing tissue stiffness and is beneficial for example to assess hepatic fibrosis [7,8]. However this method similarly suffers from susceptibility artifacts from air in the lung.

Dynamic CT (including cine-CT and four dimensional (4D) CT) images the lung in different states of expansion that could be used to derive elasticity information in contrast to static three dimensional (3D) CT. In this study, we propose to develop pulmonary nodule elastometry derived from CT images acquired at breath-hold at extreme tidal volumes. We applied deformable image registration (DIR) to the breath-hold images to determine the volume of the malignant pulmonary nodules (MPN) and a ring of surrounding lung tissue in each respiratory state. Then, elasticity was defined as the ratio of the volume change of MPN compared to the ring.

To demonstrate proof of principle that pulmonary nodule elastometry can be determined from breath-hold CT images at extreme tidal volumes, we analyzed the pre-treatment CT images of 23 patients with malignant pulmonary nodules $(\mathrm{MPN})$ treated with stereotactic ablative radiotherapy (SABR) at our institution and compared the volume change of the treated MPN with that of the surrounding lung.

\section{Methods and Materials}

\section{Patient Selection}


From all patients treated on an institutional protocol of stereotactic ablative radiotherapy (SABR) for lung tumors between November 2011 and October 2012, 23 patients had both inhale and exhale breath-hold CT scans performed at the time of simulation available for review and are included in this analysis.

\section{CT Imaging}

Deep inspiration and natural expiration breath-hold CT scans for these patients were acquired on a Discovery ST PET/CT Scanner (General Electric Medical Systems, Waukesha, WI, USA), using the following acquisition settings: $120 \mathrm{KVp}, 110-195 \mathrm{mAs}, 1.25 \mathrm{~mm}$ slice thickness, 0.97-1.36 mm pixel size, 500-700 mm display field of view. Images were reconstructed using either the built-in Bone Plus or Soft convolution kernel.

\section{Contouring}

The original treatment plans were delineated on either exhale or deep inhale based on the treating physician's

Two observers manually delineated the MPN on the opposite respiratory phase using our treatment planning software, Eclipse V11 (Varian Medical Systems, Inc., Palo Alto, CA, USA). For manual delineation, each observer applied the standard pre-defined "Lung" window-level (window width: $1324 \mathrm{HU}$, window level: $-362 \mathrm{HU}$ ) when contouring the tumors. To address inter-observer variability, the MPNs delineated by the two observers were compared to each other by calculating the Dice similarity coefficient voxel-wise $[9,10]$.

\section{Deformable Image Registration}

An intensity-based free-form deformable image registration (DIR) workflow was developed in MIM Maestro (MIM Software Inc., OH, USA) and applied on extreme tidal volumes to determine the deformation map between deep inhale and natural exhale images. The underlying algorithm parameters were inherent in the program. The similarity and smoothness criteria were combined into one energy function, which was minimized in the registration process [11]. Since the main effect of respiration on MPN is the transition of MPN whereas deformation and volume change 
of MPN is minimal [12], we separate effect of large displacement between two extreme breath-hold images from volume changes of MPN itself. Therefore the workflow starts with a tumor to tumor rigid registration with manual adjustment, followed by the DIR method which deforms the inhale (source) image to exhale (target). Rigid registration and manual adjustment yield the advantage of robustness to the MPN location.

\section{DIR Validation}

We adopted the Dice similarity coefficient to assess the quality of overlap between the manually delineated contours and deformed contours [13]. As shown in Figure S1 (a) (supplementary data), for patients with MPNs originally delineated on inhale, DIR was used to create an exhale contour. The deformed contours were then compared to the two manually delineated exhale contours using the Dice similarity metric. Similarly and as shown in Figure S1 (b) (supplementary data), for patients with MPNs originally delineated on exhale, the two observers manually delineated contours on deep inhale. Next, DIR was used to create exhale contours from the manually delineated contours on deep inhale. These deformed contours were then compared to the clinically delineated contour by Dice.

\section{Quantitative Analysis of the Deformation Map}

Because of the need for one-to-one correspondence between material points during continuous deformation, the calculated Jacobian determinant is required to be non-zero $[14,15]$. The degree of regional lung expansion is measured using the Jacobian determinant of the deformation map which is directly related to specific volume change [14].

$$
J_{n}=\frac{V_{n}+\Delta V_{n}}{V_{n}}
$$

where $V_{n}$ is the volume of voxel element $n$, and $\Delta V_{n}$ is its change in a different respiratory cycle. A value of one implies no volumetric changes, while a Jacobian determinant greater than one, or smaller than one implies local tissue expansion or local tissue contraction, respectively. It therefore follows that for a continuous deformation to be 
physically possible, the Jacobian determinant must be greater than zero. We took advantage of this fact to evaluate the accuracy of the calculated deformation map.

To validate the volumetric changes as determined by the DIR method, we used the manually delineated MPNs. The volumetric changes were expressed as the ratio of the volumes in both phases.

$$
\varphi_{\text {manual }}=\frac{V_{\text {exhale }}^{\text {MPN }}}{V_{\text {inhale }}^{M P N}}
$$

where $\varphi_{\text {manual }}$ is the manually measured volume ratio of MPN, $V_{\text {inhale }}^{M P N}$ is the volume of the MPN at deep inhale and $V_{\text {exhale }}^{M P N}$ is the volume of the MPN at natural exhale.

\section{Elasticity}

115 We derived an elasticity parameter defined as the ratio of the volumetric change of the MPN to the volumetric change of a $1 \mathrm{~cm}$ ring of lung tissue surrounding the MPN, Figure 1:

$$
d_{\varepsilon}=\frac{\left|\varphi_{D I R}{ }_{M N N}-1\right|}{\mid \varphi_{D I R} \text { Ring }^{-1} \mid}
$$

where $\varphi_{D I R}$ MPN is the calculated volumetric ratio (exhale/inhale) of MPN and $\varphi_{D I R_{R i n g}}$ is the calculated volumetric ratio (exhale/inhale) of a $1 \mathrm{~cm}$ ring around the MPN. As stated above, in some cases the clinically delineated volume was on the exhale $\mathrm{CT}$ and in some it was on the inhale $\mathrm{CT}$, but in all cases the volume ratios $\left(\varphi_{D I R}\right)$ were taken to be the exhale to inhale ratio. The normalization to the $1 \mathrm{~cm}$ ring around the MPN, was introduced to remove the effect of MPN location and amount of motion in the lung, as both the MPN and the $1 \mathrm{~cm}$ ring surrounding lung tissue undergo the same force. The choice of using a $1 \mathrm{~cm}$ ring surrounding the MPN's is somewhat arbitrary, however based on the size of the tumors, this ring size allows equal contribution of lung tissue and MPN into the calculated elasticity parameter. The calculated elasticity bigger than one $\left(d_{\varepsilon}>1\right)$ shows that the volume changes of MPN is more than that of the ring and the calculated elasticity smaller than one $\left(d_{\varepsilon}<1\right)$ shows that the volume changes of MPN is less than that of the ring. 


\section{Statistical Analysis}

130 An equivalence test was performed to assess that the manually measured volume ratio was equivalent to the DIR calculated volume ratio of MPN. A paired $t$ test was performed to compare the volume change of the normal tissue and the tumor tissue with both tissue samples coming from the same patient. All analyses were performed using SAS version 9.4 (SAS Institute Inc., Cary, NC, USA).

\section{$135 \quad$ Results}

\section{Patient and Tumor Characteristics}

We have included 23 lung cancer patients with primary non-small cell lung cancer (NSCLC) or limited lung metastases that were treated at our institution. The patient and tumor characteristics are given in Table 1.

\section{DIR Validation}

For all patients, the Dice similarity coefficient between the delineated tumors by observer 1 and that of observer 2 is $0.81 \pm 0.1$, whereas the Dice value is $0.79 \pm 0.07$ and $0.79 \pm 0.06$ between DIR and observer 1 and DIR and observer 2, respectively. Figure 2 shows an example of both delineated and deformed contours for a representative patient as well as the distribution of the calculated Dice similarity for all patients. For all patients we calculated the Jacobian

145 determinant of the deformation map and validated the calculated volume ratio of MPN from the deep inhale phase to the natural exhale phase against the manual contouring. The calculated Jacobian determinant of the deformation map within the lung is always greater than zero. We performed an equivalence test with the indifference zone defined as $[-0.25,0.25]$. The mean of the differences of the paired values was 0.0293 with a $95 \%$ confidence interval of [0.0176, 0.0761]. The 95\% confidence interval falls completely within the indifference zone thus proving 150 equivalence $(\mathrm{p}<0.0001)$. The average difference between the manually measured volume ratio of MPN $\left(\varphi_{\text {manual }}\right)$ and calculated volume ratio value via DIR $\left(\varphi_{D I R_{M P N}}\right)$ was $0.02 \pm 0.15$. Figure 3 shows the calculated volume ratio by DIR versus manually measured volume ratio of MPN for all patients as well as the distribution of the difference 
between the calculated volume ratio and manually measured volume ratio of MPN for all patients which mainly concentrated around zero.

\section{MPN Elasticity}

For all patients in our study, the mean and standard deviation of the calculated volume ratio of the MPN and that of the $1 \mathrm{~cm}$ ring surrounding lung tissue was $0.91 \pm 0.18$ and $0.75 \pm 0.13$, respectively. The mean and standard deviation of the difference between the calculated volume ratio of the MPN $\left(\varphi_{D I R_{M P N}}\right)$ with that of $1 \mathrm{~cm}$ ring surrounding

160 lung tissue $\left(\varphi_{\text {DIR Ring }}\right)$ was $0.16 \pm 0.15(\mathrm{p}<0.0001)$. Figure 4 (a) shows the calculated volume ratio of MPN and $1 \mathrm{~cm}$ ring surrounding lung tissue for all patients. The volume ratio is mainly distributed below the unity line demonstrating that the volume ratio of the MPNs are less than that of the $1 \mathrm{~cm}$ ring surrounding lung tissue.

Finally, based on our definition of the elasticity parameter in Eq. 3, the mean and standard deviation of the calculated elasticity $\left(d_{\varepsilon}\right)$ of the MPN was $0.78 \pm 1.1$, and the median value was 0.58 . Figure 4 (b) shows the 165 distribution of the calculated elasticity for all patients.

Table 2 (supplementary data) shows tumor size in inhale and exhale, as well as the calculated volume ratio of MPN by DIR, the calculated elasticity, histology, and lobar location. The median elasticities for lower and upper/middle lobe MPNs are 0.53 and 0.61 , respectively.

\section{Discussion}

We have designed and developed a method to determine the elasticity of MPNs based on deformable image registration of breath-hold CT images. Various deformable image registration methods [12,16-19] and mechanical indices [20] have been surveyed to calculate the most accurate and robust deformation map as well as differentiation metric, respectively. We developed a workflow that processed breath-hold images at extreme tidal volumes and calculated the volume change and the elasticity of MPNs. 
We have demonstrated robust performance of the DIR method such that the difference between DIR and each of the observers is comparable to the inter-observer difference as assessed by Dice similarity. Our method can measure the volume ratio of MPNs, which can be either expansion or contraction. The accuracy of this measurement is demonstrated by the small difference between the manually measured volume ratios to that of the DIR method $(0.02 \pm 0.15)$.

Based on the presented results in Figure 4 (a), $0<\varphi_{D_{\text {IR Ring }}}<\varphi_{\text {DIR } M P N}<1$ which demonstrates that MPNs have a lesser volume change than the $1 \mathrm{~cm}$ ring surrounding lung tissue during exhalation. Thus, one expects $d_{\varepsilon}=$ $\frac{\left|\varphi_{\text {DIRMPN }}-1\right|}{\left|\varphi_{D_{\text {IR }} \text { Ring }}-1\right|}<1$ which has been shown in Figure $4(\mathrm{~b}),\left(d_{\varepsilon}=0.78 \pm 1.1\right)$. This demonstrated that MPNs have different characteristics in terms of elasticity compared to the surrounding lung tissue during respiration.

185 Different parts of the lung have different motion amplitudes and volume changes, which makes the calculated volume ratio of MPNs location-dependent. However the formulated elasticity is not location-dependent because the normalization of the volume change of MPN to that of the $1 \mathrm{~cm}$ ring surrounding lung tissue should remove the effect of MPN location, as both the MPN and the $1 \mathrm{~cm}$ ring surrounding lung tissue undergo the same force, irrespective of their location. Therefore, it allows us to compare the elasticity of two different MPNs in two different 190 locations of lung. This is supported by the similar median values of the elasticity parameter for lower vs. upper/middle lobe MPNs.

Our study does have certain limitations. First, our method was applied to a small number of patients, and focused only on proven MPNs, excluding benign nodules. The imaging protocol for all patients was not completely uniform which resulted in different image resolutions that can impact the analysis. However we employed image cubic spline 195 interpolation so that DIR was applied to images of the same resolution.

In addition, there are many possible expressions for the definition of elasticity, all of which are only surrogates for physical elasticity which requires knowledge of applied forces that cannot be determined from images. While a value greater or less than 1 indicates MPN volume ratio greater or less than the surrounding ring, as seen in Figure 4, this results in a distribution that is skewed. An alternative formulation such as $\left(\log \frac{\varphi_{D I R M P N}}{\varphi_{D I R_{R I n g}}}\right)$ might be more 
physically intuitive since expansion or compression would have symmetrical effects but with opposite sign about zero. Such formulations may be helpful to explore in future studies.

To the best of our knowledge, this is the first study to calculate the elasticity of MPN from dynamic thoracic CT images. This provides the technical framework for testing the hypothesis that MPN and benign pulmonary nodule differ in elasticity. This difference may come from increased interstitial fluid pressure in tumors which can change the elasticity of MPN prior to any changes in morphological features. Studies have shown that most solid tumors have increased interstitial fluid pressure. This has been shown for breast carcinoma [2,3], metastatic melanoma [4,5], head and neck carcinoma [6], and colorectal carcinoma [2], with values as high as $60 \mathrm{mmHg}$. The tumor interstitial fluid pressure is uniform throughout the center of the tumor and drops steeply in its periphery.[21-23]. The mechanisms that determine the increased tumor interstitial fluid pressure are not fully understood, but are 210 thought to involve blood-vessel leakiness, lymphatic vessel abnormalities, interstitial fibrosis and a contraction of the interstitial space mediated by stromal fibroblasts, all of which are hallmarks of cancer $[24,25]$.

Since a limitation of current CT based screening for lung cancer is distinguishing malignant from benign nodule [26], our method could impact clinical practice by increasing the specificity of CT-based lung cancer screening. In addition CT elastometry may predict tumor aggressiveness that could potentially be used in multifocal lung cancer

215 patients to ascertain and treat the most aggressive lesions first. Ultimately it may even be helpful to distinguish aggressiveness of different tumor regions to guide radiation therapy dose painting or adaptive boost design.

Future directions include applying our analysis to both proven malignant and benign nodules, and ultimately may differentiate the aggressiveness of tumors or tumor regions to aid radiation therapy target selection or dose painting [27]. We will also evaluate nodule deformation homogeneity using strain tensors map, to determine whether this 220 information could aid in distinguishing between malignant and non-malignant nodules. In addition we will attempt to extend our method to 4D CT images routinely acquired for radiotherapy simulation.

In conclusion, we developed a noninvasive method based on DIR of inhale/exhale breath-hold images to determine MPN elasticity. 


\section{References}

1. Sayyouh M, Vummidi DR, Kazerooni EA. Evaluation and management of pulmonary nodules: state-of-theart and future perspectives. Expert opinion on medical diagnostics 2013;7:629-44.

2. Less JR, Posner MC, Boucher Y, Borochovitz D, Wolmark N, Jain RK. Interstitial hypertension in human breast and colorectal tumors. Cancer Research 1992;52:6371-4.

2303 3. Nathanson SD, Nelson L. Interstitial fluid pressure in breast cancer, benign breast conditions, and breast parenchyma. Ann Surg Oncol 1994;1:333-8.

4. Boucher Y, Kirkwood JM, Opacic D, Desantis M, Jain RK. Interstitial hypertension in superficial metastatic melanomas in humans. Cancer Research 1991;51:6691-4.

5. Curti BD, Urba WJ, Alvord WG, et al. Interstitial pressure of subcutaneous nodules in melanoma and

235 lymphoma patients: changes during treatment. Cancer Research 1993;53:2204-7.

6. Gutmann R, Leunig M, Feyh J, et al. Interstitial hypertension in head and neck tumors in patients: correlation with tumor size. Cancer Research 1992;52:1993-5.

7. Yin M, Talwalkar JA, Glaser KJ, et al. Assessment of Hepatic Fibrosis With Magnetic Resonance Elastography. Clinical Gastroenterology and Hepatology 2007;5:1207-13.

240 8. Venkatesh SK, Yin M, Ehman RL. Magnetic resonance elastography of liver: Technique, analysis, and clinical applications. Journal of Magnetic Resonance Imaging 2013;37:544-55.

9. Dice LR. Measures of the Amount of Ecologic Association Between Species. Ecology 1945;26:297-302.

10. Riegel AC, Chang JY, Vedam SS, Johnson V, Chi P-CM, Pan T. Cine Computed Tomography Without Respiratory Surrogate in Planning Stereotactic Radiotherapy for Non-Small-Cell Lung Cancer. International Journal 245 of Radiation Oncology*Biology*Physics 2009;73:433-41.

11. Nie K, Chuang C, Kirby N, Braunstein S, Pouliot J. Site-specific deformable imaging registration algorithm selection using patient-based simulated deformations. Medical Physics 2013;40:041911-1.

12. Wu J, Lei P, Shekhar R, Li H, Suntharalingam M, D'Souza WD. Do Tumors in the Lung Deform During Normal Respiration? An Image Registration Investigation. International Journal of Radiation Oncology*Biology*Physics 2009;75:268-75. 
13. Liu X, Saboo RR, Pizer SM, Mageras GS. A shape-navigated image deformation model for 4D lung respiratory motion estimation. IEEE Int Symp Biomed Imaging:2009:875-8.

14. Reinhardt JM, Ding K, Cao K, Christensen GE, Hoffman EA, Bodas SV. Registration-based estimates of local lung tissue expansion compared to xenon-CT measures of specific ventilation. Medical Image Analysis $2008 ; 12: 752-63$.

15. Negahdar M, Amini AA. Regional lung strains via a volumetric mass conserving optical flow model. IEEE International Symposium on Biomedical Imaging (ISBI): From Nano to Macro; Barcelona, Spain:2012.

16. Negahdar M, Amini AA. Tracking planar lung motion in 4D CT with optical flow: validations and comparison of global, local, and local-global methods. Medical Imaging 2010: Biomedical Applications in

260 Molecular, Structural, and Functional Imaging; San Diego, CA, USA 2010;7626-74.

17. Chaojie Z, Xiuying W, Jinhu C, Yong Y, Dagan F. Deformable registration model with local rigidity preservation for radiation therapy of lung tumor. Image Processing (ICIP), 2012 19th IEEE International Conference on:2012:1673-6.

18. van Dam IE, van Sörnsen de Koste JR, Hanna GG, Muirhead R, Slotman BJ, Senan S. Improving target 265 delineation on 4-dimensional CT scans in stage I NSCLC using a deformable registration tool. Radiotherapy and Oncology 2010;96:67-72.

19. Speight R, Sykes J, Lindsay R, Franks K, Thwaites D. The evaluation of a deformable image registration segmentation technique for semi-automating internal target volume (ITV) production from 4DCT images of lung stereotactic body radiotherapy (SBRT) patients. Radiotherapy and Oncology 2011;98:277-83.

$27020 . \quad$ Negahdar M, Dunlap N, Zacarias A, Eivelek AC, Woo SY, Amini AA. Strain as a novel index of regional pulmonary function from thoracic 4D CT images: in-vivo validation with tomographic SPECT ventilation and perfusion. Medical Imaging 2013: Biomedical Applications in Molecular, Structural, and Functional Imaging; Orlando, FA, USA 2013;8672-38.

21. Boucher Y, Baxter LT, Jain RK. Interstitial pressure gradients in tissue-isolated and subcutaneous tumors: 275 implications for therapy. Cancer Research 1990;50:4478-84.

22. DiResta GR, Lee J, Larson SM, Arbit E. Characterization of neuroblastoma xenograft in rat flank. I. Growth, interstitial fluid pressure, and interstitial fluid velocity distribution profiles. Microvasc Research 1993;46:158-77. 
23. Eikenes L, Bruland ØS, Brekken C, Davies CdL. Collagenase increases the transcapillary gradient and improves the uptake and distribution of monoclonal antibodies in human osteosarcoma xenografts. Cancer Research 2004;64:4768-73.

24. Heldin $\mathrm{CH}$, Rubin K, Pietras K, Ostman A. High interstitial fluid pressure - an obstacle in cancer therapy. Nat Rev Cancer 2004;4:806-13.

25. Sarntinoranont M, Rooney F, Ferrari M. Interstitial stress and fluid pressure within a growing tumor. Ann 285 Biomed Eng 2003;31:327-35.

26. Aberle DR, Adams AM, Berg CD, et al. Reduced lung-cancer mortality with low-dose computed tomographic screening. N Engl J Med 2011;365:395-409.

27. Maldonado F, Boland JM, Raghunath S, et al. Non-invasive Characterization of the Histopathologic Features of Pulmonary Nodules of the Lung Adenocarcinoma Spectrum using Computer Aided Nodule Assessment 290 and Risk Yield (CANARY) - a Pilot Study. Journal of thoracic oncology : official publication of the International Association for the Study of Lung Cancer 2013;8:452-60. 


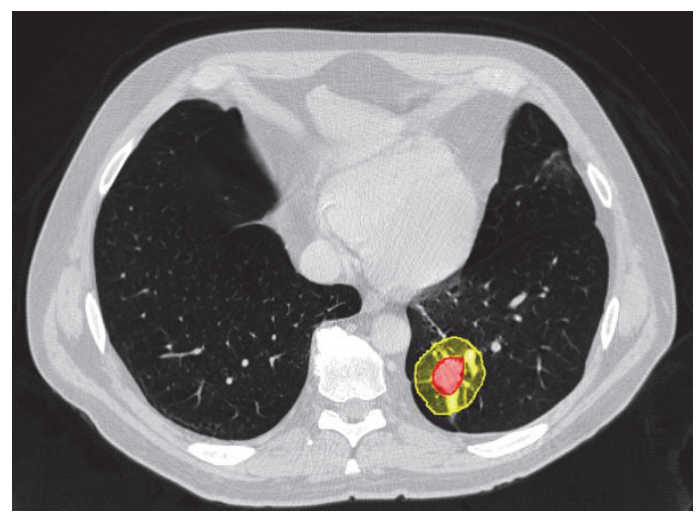

Deep Inhale CT

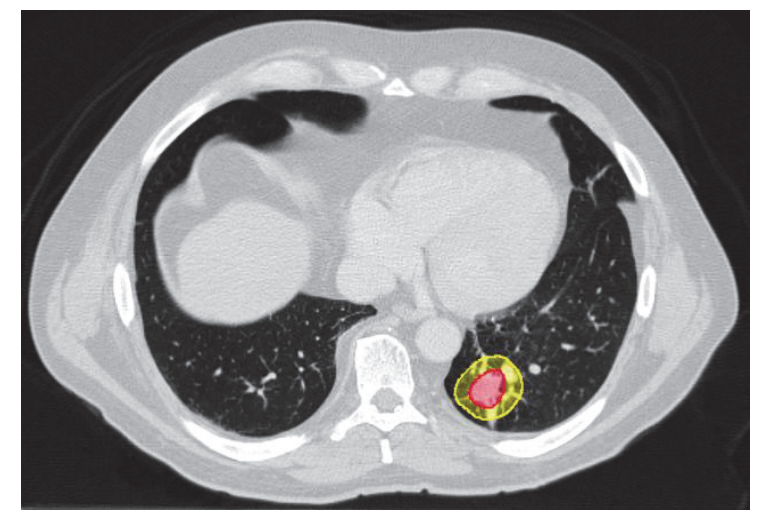

Natural Exhale CT with Deformed Contours

Figure 1. An axial view of a representative patient; delineated MPN (inner circle) and $1 \mathrm{~cm}$ ring of the surrounding lung (outer circle) for deep inhale (left column) and deformed MPN and ring contours superimposed on exhale image (right column) for the purpose of elasticity calculation. 

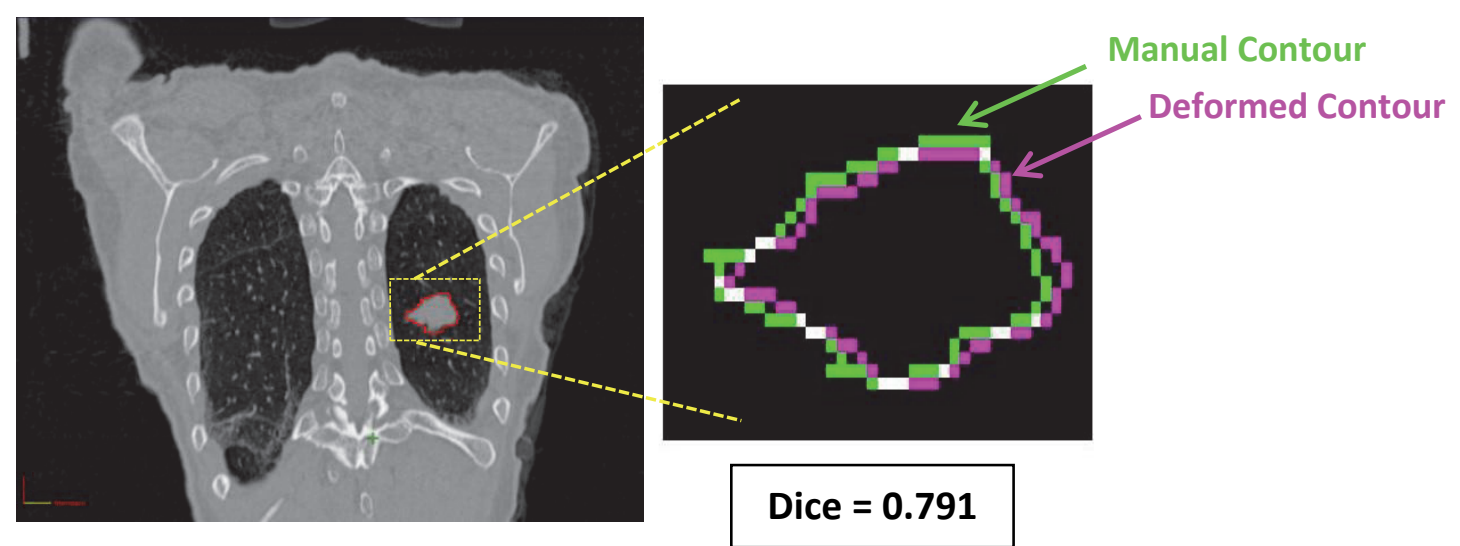

(a)

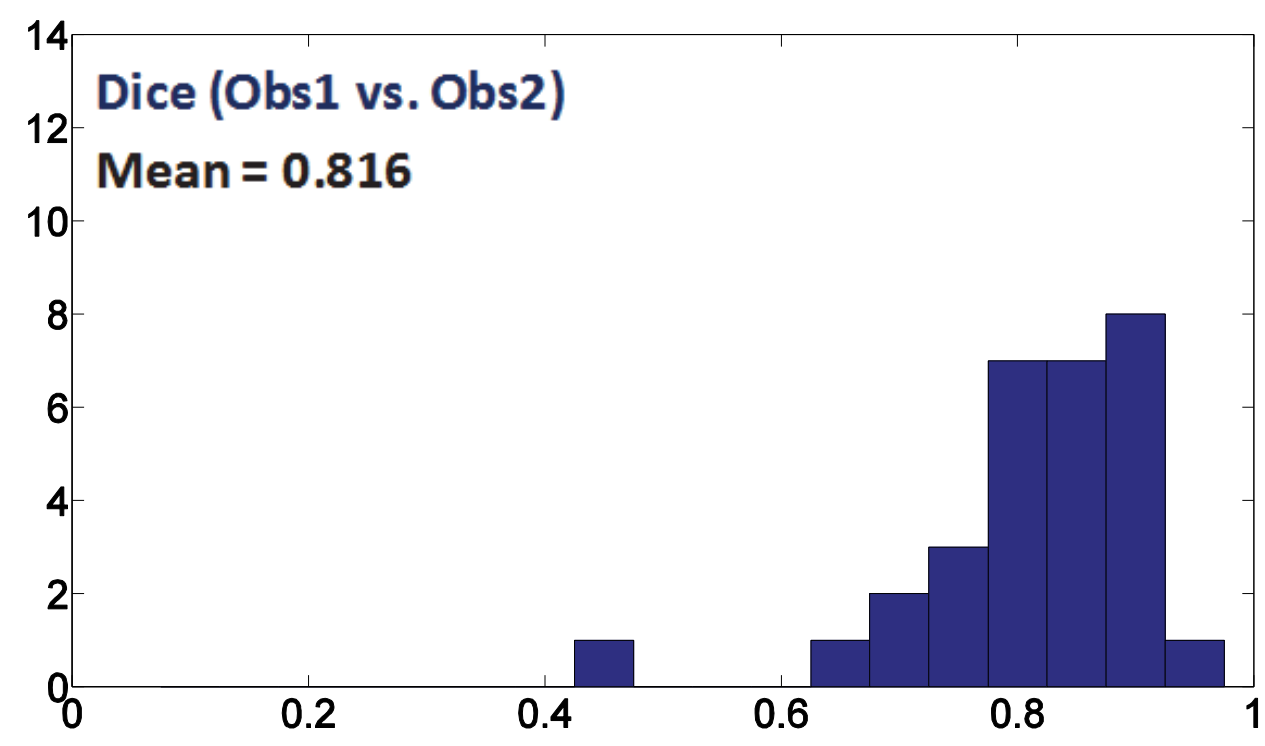

(b) 


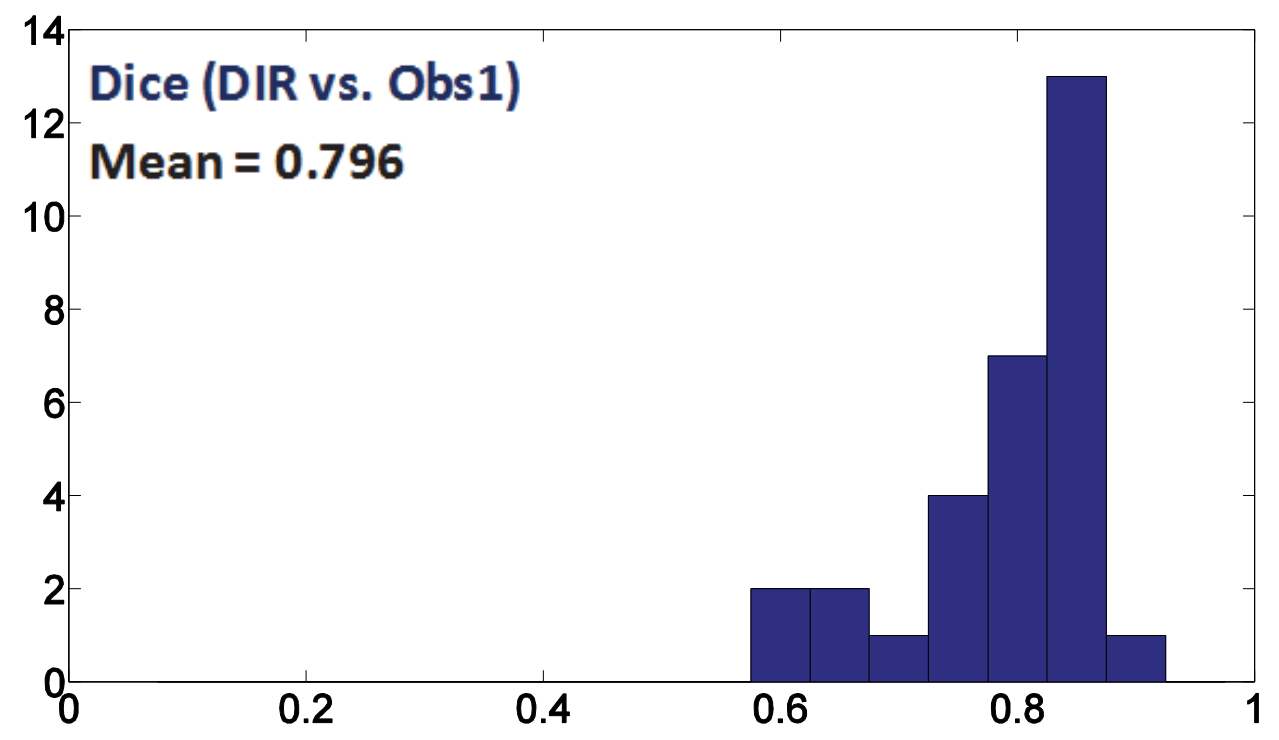

(c)

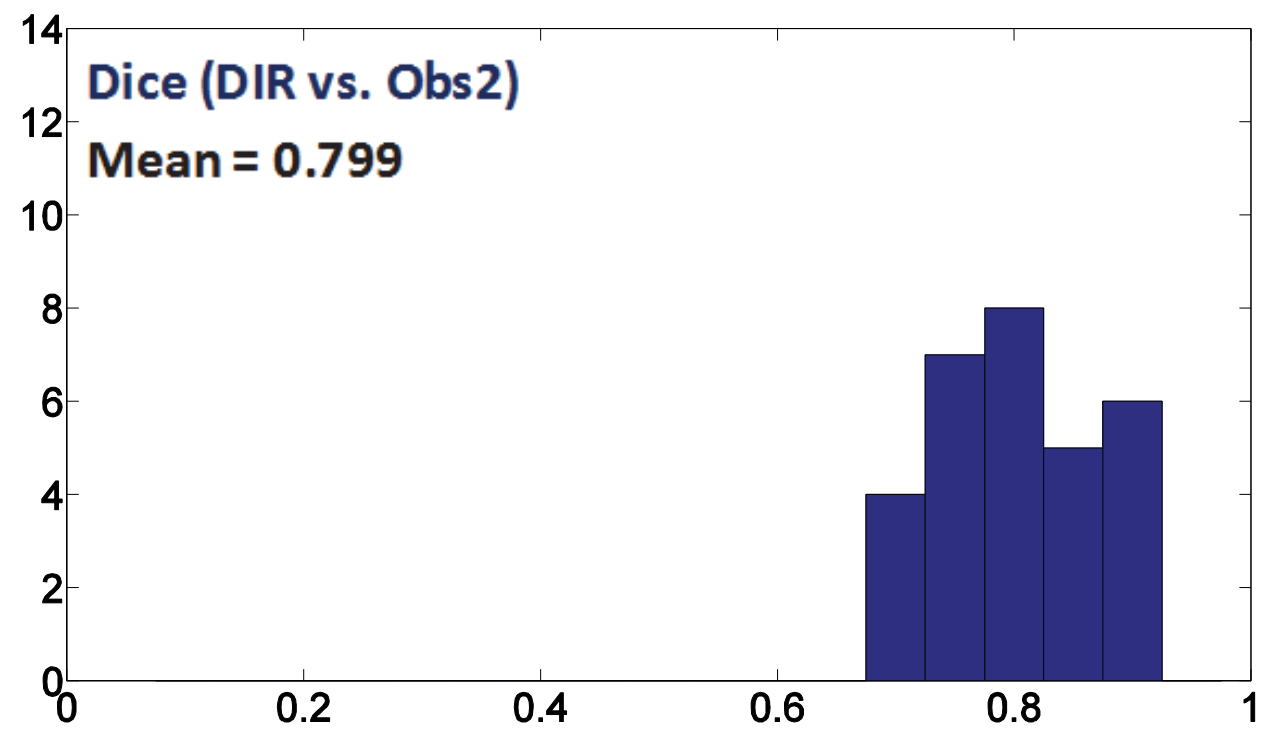

(d)

Figure 2. (a) A coronal view of a manually delineated tumor in exhale of a representative patient; delineated MPN in exhale (green) and delineated MPN in inhale deformed into the exhale (magenta), Dice similarity for this patient is 0.791 . (b) Distribution of the calculated Dice similarity metric between the manually delineated MPN by first observer with that of second observer. (c) Distribution of Dice similarity between the delineated MPN in inhale deformed into the exhale phase by DIR with the manually delineated MPN by first observer in exhale. (d) Distribution of Dice similarity between the delineated MPN in inhale deformed into the exhale phase by DIR with the manually delineated MPN by second observer in exhale. Based on dice similarity, we conclude that DIR compares favorably to manual contouring within inter-observer differences. 

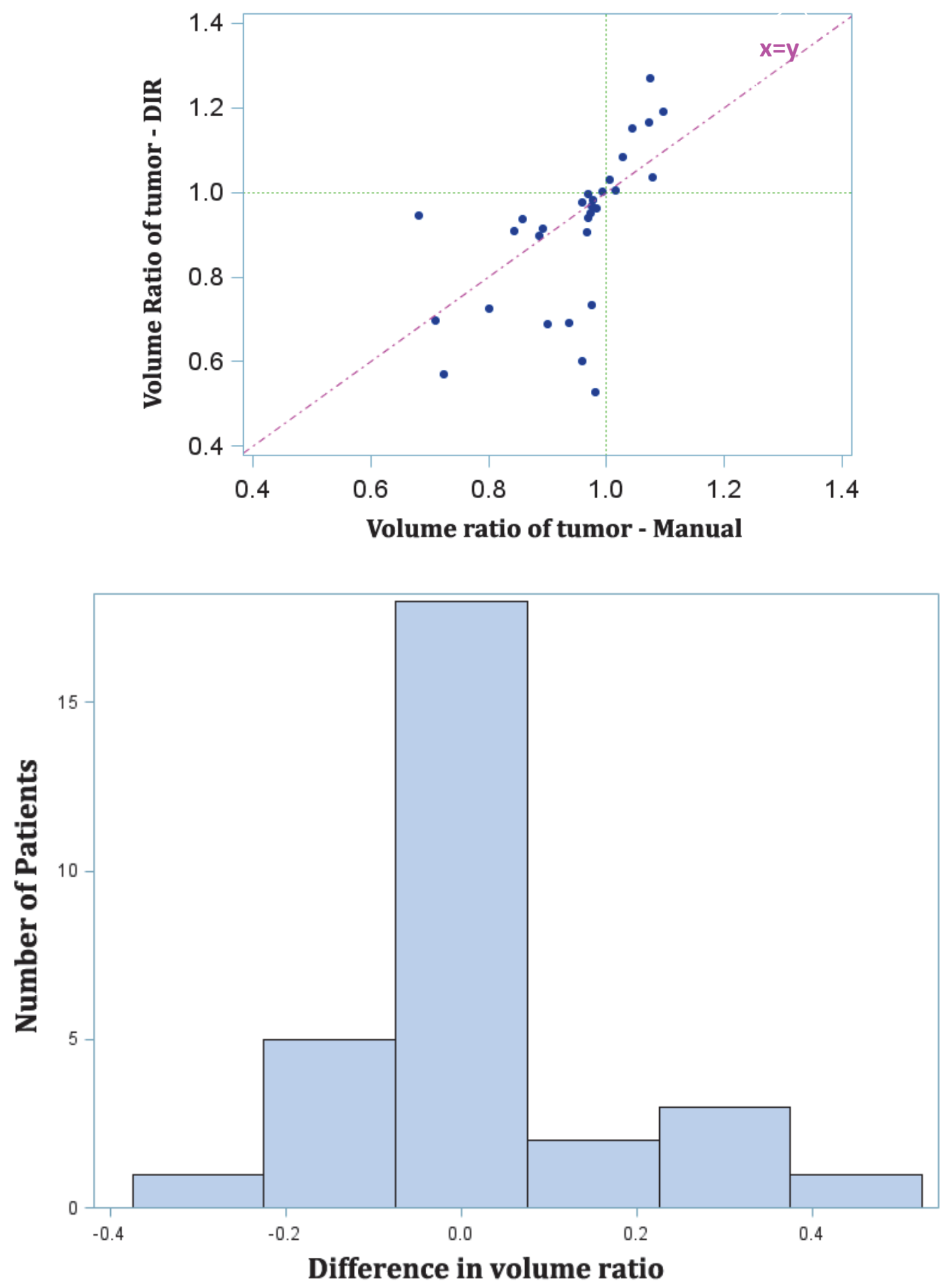

Figure 3. (Up) Calculated volume ratio of MPN versus manually measure of volume ratio of MPN (Equation (2)) for all 30 patients from inhale to exhale. Green dashed line shows unity Jacobian value equivalent to no volume changes. Red line represents the identity line. (Bottom) Distribution of the difference between the calculated volume ratio by DIR from inhale to exhale with manually measured volume ratio of MPN $(0.02 \pm 0.15)$. 


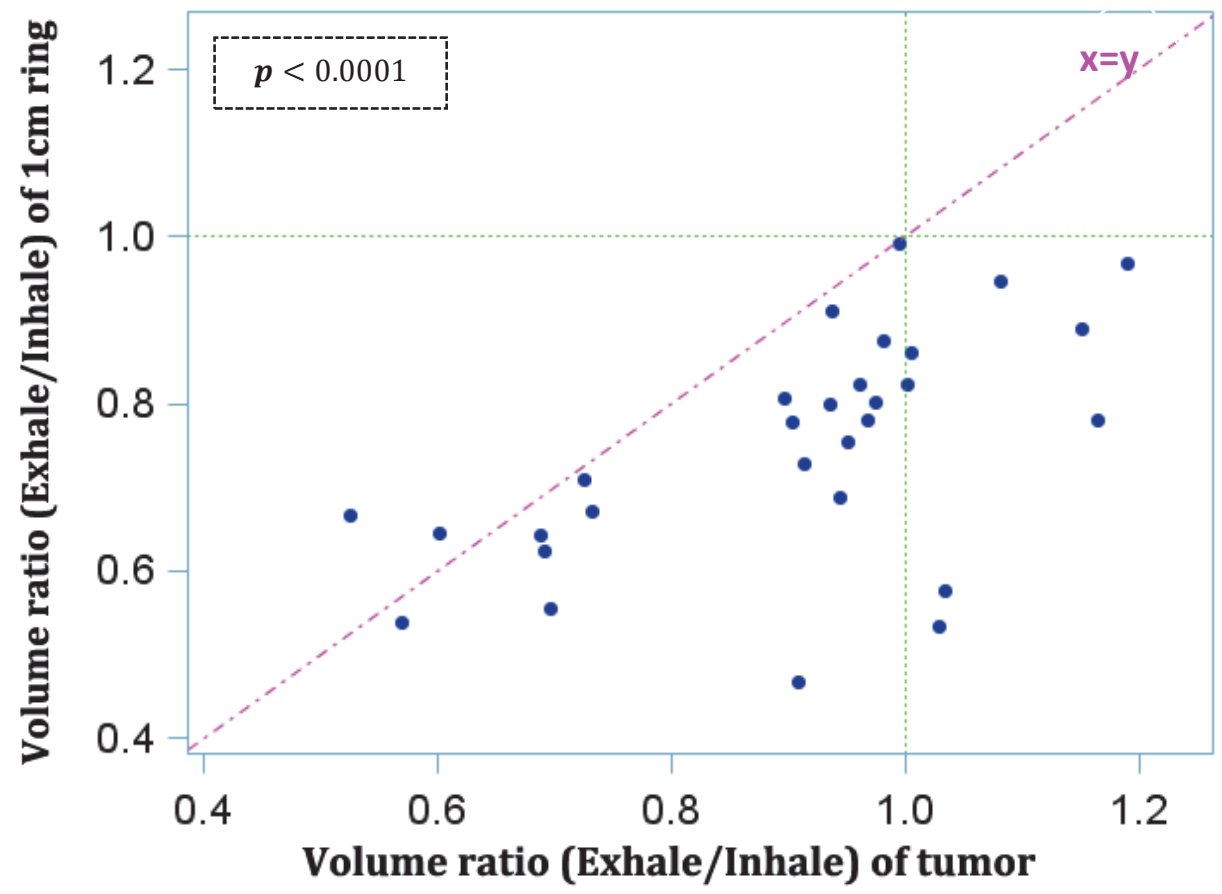

(a)

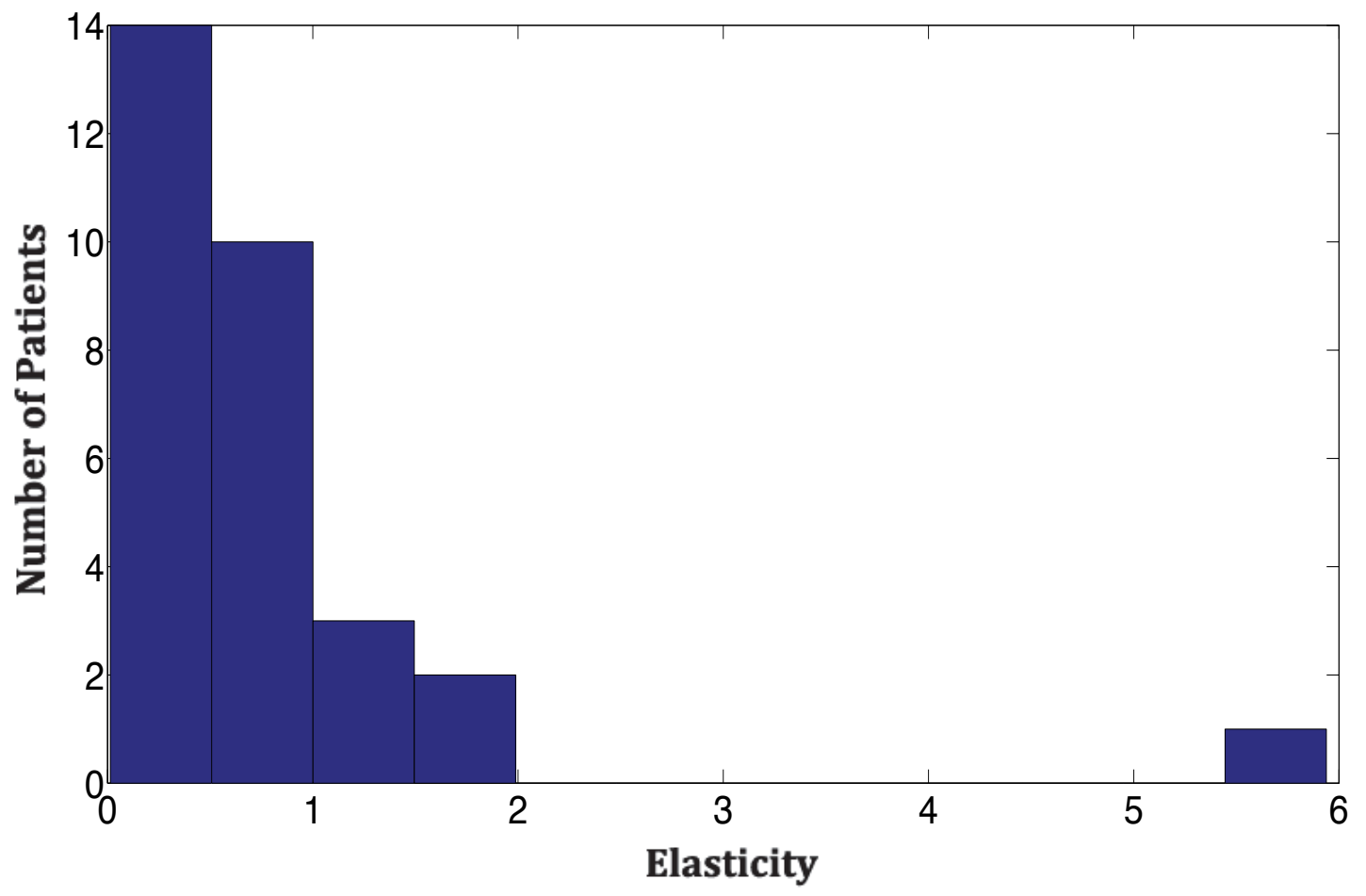

(b)

Figure 4. (a) Calculated volume ratio of $1 \mathrm{~cm}$ ring of surrounding lung $(0.75 \pm 0.13)$ versus that of tumor $(0.91 \pm 0.18)$ for all patients. Green dashed line shows unity Jacobian value equivalent to no volume changes. Red line represents the identity line. (b) Distribution of the calculated elasticity by Equation (3) from inhale to exhale $(0.78 \pm 1.1)$. 
Table 1. Patient and tumor characteristics.

\begin{tabular}{|c|c|}
\hline Characteristic & Value (\%) \\
\hline All Patients & 23 \\
\hline \multicolumn{2}{|l|}{ Gender } \\
\hline Male & $13(57)$ \\
\hline Female & $10(43)$ \\
\hline \multicolumn{2}{|l|}{ Age, $y$} \\
\hline Median & 66 \\
\hline Range & $35-94$ \\
\hline Tumors & 30 \\
\hline Primary/Secondary & $18 / 12$ \\
\hline \multicolumn{2}{|l|}{ Histology } \\
\hline Adenocarcinoma & 21 \\
\hline Squamous & 9 \\
\hline \multicolumn{2}{|c|}{ Radius of Tumors, cm (treatment planning) } \\
\hline Median & 1 \\
\hline Range & $0.3-2.1$ \\
\hline \multicolumn{2}{|c|}{ Volume of Tumors, $\mathrm{cm}^{3}$ (treatment planning) } \\
\hline Median & 4.21 \\
\hline Range & $0.11-38.8$ \\
\hline \multicolumn{2}{|c|}{ Volume of Tumors in opposite phases, $\mathrm{cm}^{3}$ (Obs 1) } \\
\hline Median & 4.3 \\
\hline Range & $0.13-39.62$ \\
\hline \multicolumn{2}{|c|}{ Volume of Tumors in opposite phases, $\mathrm{cm}^{3}$ (Obs 2) } \\
\hline Median & 4.29 \\
\hline Range & $0.13-39.74$ \\
\hline
\end{tabular}

